\title{
ON OPERATOR-VALUED STOCHASTIC INTEGRALS
}

\author{
BY HUI-HSIUNG $\mathrm{KUO}^{1}$ \\ Communicated by Harry Kesten, June 7, 1972
}

1. Introduction. The purpose of this note is to announce some theorems concerning the operator-valued stochastic integrals which arise naturally from the study of the regularity properties of solutions of stochastic integral equations. Proofs and detailed discussion will be given in [7]. Let $B^{*} \subset H \subset B$ be an abstract Wiener space [1] and $W(t)$ a Wiener process in $B$. Various stochastic integrals associated with $W(t)$ were studied and an infinite-dimensional version of Ito's formula [2] was proved in [5]. This formula was used to connect the solution of a stochastic integral equation with the corresponding heat equation [8]. In [6] we proved another version of Ito's formula which was used to construct diffusion processes, in particular, a Brownian motion, in a RiemannWiener manifold. We present here a third version of Ito's formula and use it to prove an infinite-dimensional analogue of a formula on p. 58 of McKean's book [9]. An operator-valued stochastic integral has been studied by Kannan and Bharucha-Reid [4]. However, there appears to be no relation between their work and ours in this paper.

2. Notation and definitions. Let $X$ and $Y$ be two real Banach spaces. $L^{n}(X ; Y)$ denotes the Banach space of all continuous $n$-linear maps from $X^{n}$ into $Y$ with the usual norm $\|\cdot\|_{X^{n} ; Y} \cdot L^{1}$ will be written as $L . L^{n-1}\left(X ; X^{*}\right)$ will be identified as $L^{n}(X ; R)$ in a well-known way. $L_{(2)}^{2}(H ; R)\left(\equiv L_{(2)}(H ; H)\right)$ denotes the Hilbert space of all Hilbert-Schmidt operators of $H$ with H-S-norm $\|\cdot\|_{2}=\langle\langle,\rangle\rangle^{1 / 2}$. We have the relation $L^{2}(B ; R) \subset L_{(2)}^{2}(H ; R)$.

DEFINITION. Let $K$ be a Hilbert space with inner product (,). A continuous bilinear map $S$ from $H \times H$ into $K$ is said to be of trace class type if (i) for each $x \in K, S_{x}$ is a trace class operator of $H$, where $S_{x}(\cdot, \cdot)=$ $(S(\cdot, \cdot), x)$ and (ii) the functional $x \rightarrow \operatorname{trace} S_{x}$ is continuous. $\mathscr{S}(H ; K)$ denotes the space consisting of all such continuous bilinear maps.

Let $S$ be of trace class type. Then there is a unique element, denoted by TRACE $S$, of $K$ such that (TRACE $S, x)=$ trace $S_{x}$ for all $x \in K$. Note that $L^{2}\left(B ; L\left(B, B^{*}\right)\right) \subset \mathscr{S}\left(H ; L_{(2)}(H ; H)\right)$.

AMS (MOS) subject classifications (1970). Primary 60H05, 60H20.

Key words and phrases. Abstract Wiener space, Ito's formula, operator-valued process, trace class type, Hilbert-Schmidt type, stochastic differential equation, GirsanovSkorokhod-McKean's formula.

${ }^{1}$ This research was supported in part by NSF grant GU-3784. 
Definition. Let $T \in L^{3}(H ; R)$. Define $\tilde{T} \in L(H ; L(H, H))$ by $\tilde{T}(h)=$ $T(h, \cdot, \cdot) . T$ is said to be of Hilbert-Schmidt type if (i) $\widetilde{T}(H) \subset L_{(2)}(H, H)$ and (ii) $\tilde{T}$ is a Hilbert-Schmidt operator from $H$ into $L_{(2)}(H, H) . L_{(2)}^{3}(H ; R)$ denotes the space consisting of all such $T$ 's.

Define $\|T\|_{2}$ to be the H-S-norm of $\tilde{T}$. Clearly, $\|T\|_{2}^{2}=\sum_{i, j, k} T\left(e_{i}, e_{j}, e_{k}\right)^{2}$ for any O.N.B. $\left\{e_{k}\right\}_{k=1}^{\infty}$ of $H . L_{(2)}^{3}(H ; R)$ is a Hilbert space. Moreover, we have the relation $L^{3}(B ; R) \subset \mathscr{S}(H ; H) \subset L_{(2)}^{3}(H ; R)$.

3. Theorems. As in [5] we assume the following on the abstract Wiener space $B^{*} \subset H \subset B$ : There exists a sequence $Q_{n}$ of finite-dimensional projections such that (i) $Q_{n}(B) \subset B^{*}$ and (ii) $Q_{n}$ converges strongly to the identity both in $B$ and in $H$.

$L(H, H)$ has three topologies, namely, the uniform topology, strong topology and weak topology. However, the Borel fields corresponding to them are all the same. This can be shown by a similar argument used in [3]. Therefore, when we talk about the measurability of a random variable (resp. a process) with values in (resp. state space) $L(H, H)$ there is no need to specify the Borel field. We have the same situation for $L\left(B ; L\left(B, B^{*}\right)\right) \approx$ $L^{3}(B ; R)$. Let $\xi(t)$ be a simple nonanticipating (with respect to the Wiener process $W(t))$ process with state space $L^{3}(B ; R)$. Suppose $\xi$ has jumps at $0<t_{1}<\cdots<t_{n}$. Define

$$
I_{\xi}(t)=\sum_{k=0}^{j-1} \tilde{\xi}\left(t_{k}\right)\left(W\left(t_{k+1}\right)-W\left(t_{k}\right)\right)+\tilde{\xi}\left(t_{j}\right)\left(W(t)-W\left(t_{j}\right)\right),
$$

if $t_{j} \leqq t<t_{j+1}$. Here $t_{0}=0$ and $t_{n+1}=\infty$. We will regard $I_{\xi}(t)$ as a process with state space $L(H, H)$ via the inclusions $L\left(B ; B^{*}\right) \subset L_{(2)}(H, H)$ $\subset L(H, H)$.

THEOREM 1. For every nonanticipating process $\xi(t)$ with state space $L^{3}(B ; R)$ such that $\int_{0}^{\tau}\|\xi(t)\|_{B^{3} ; R}^{2} d t<\infty$ a.s. for each $0<\tau<\infty$, we can determine a stochastic process

$$
I_{\xi}(t) \equiv \int_{0}^{t} \xi(s) d W(s)
$$

satisfying the following properties:

(i) $I_{\xi}$ has continuous sample paths (uniform topology for $L(H, H)$ );

(ii) $I_{\xi}$ is a martingale;

(iii) $\operatorname{prob}\left\{\sup _{0 \leqq t \leqq}\left\|I_{\xi}(t)\right\|_{2}>\delta\right\} \leqq \delta^{-2} E\left\{\left\|I_{\xi}(\tau)\right\|_{2}^{2}\right\}$;

(iv) $E\left\{I_{\xi}(t)\right\}=0$ and $E\left\{\left\|I_{\xi}(t)\right\|_{2}^{2}\right\}=E \int_{0}^{t}\|\xi(s)\|_{2}^{2} d s$;

(v) $I_{\alpha \xi_{1}+\beta \xi_{2}}=\alpha I_{\xi_{1}}+\beta I_{\xi_{2}}$

(vi) $I_{\xi}$ is nonanticipating.

Let $S \in L(L(H ; H) ; L(H ; H))$ and $T \in L^{3}(H ; R)$. Define $S \triangle T \in L^{3}$ $(H ; R)$ by $(S \triangle T)^{\sim}=S \circ \widetilde{T}$. Note that if $L\left(B ; B^{*}\right)$ is invariant under $S$ and $T \in L^{3}(B ; R)$ then $S \triangle T \in L^{3}(B ; R)$. Note also that if $T \in L^{3}(B ; R)$ and $U \in L^{2}(L(H ; H) ; L(H ; H))$ is such that $U\left(L\left(B ; B^{*}\right) \times L\left(B ; B^{*}\right)\right) \subset$ 
$L\left(B ; B^{*}\right)$ then $U \circ[T \times T] \in L^{2}\left(B ; L\left(B ; B^{*}\right)\right) \subset \mathscr{S}\left(H ; L_{(2)}(H ; H)\right)$ so that we have TRACE $U \circ[T \times T] \in L_{(2)}(H ; H)$.

TheOREM 2 (Ito's Formula). Let $\theta$ be a $C^{2}$-map from $L(H ; H)$ into itself such that $\theta^{\prime}(S)\left(L\left(B ; B^{*}\right)\right) \subset L\left(B ; B^{*}\right)$ and $\theta^{\prime \prime}(S)\left(L\left(B ; B^{*}\right) \times L\left(B ; B^{*}\right)\right)$ $\subset L\left(B ; B^{*}\right)$ for all $S \in L(H ; H)$. If $d X(t)=\xi(t) d W(t)+\zeta(t) d t$, where $\xi(t)$ is a nonanticipating process in $L^{3}(B ; R)$ such that $\int_{0}^{\tau}\|\xi(t)\|_{B ; R}^{2} d t<\infty$ a.s. for each $0<\tau<\infty$ and $\zeta(t)$ is a nonanticipating process in $L(H ; H)$ with $\int_{0}^{\tau}\|\zeta(t)\|_{H, H} d t<\infty$ a.s. for each $0<\tau<\infty$ then

$$
\begin{aligned}
d \theta(X(t))= & \theta^{\prime}(X(t)) \triangle \xi(t) d W(t) \\
& +\left\{\theta^{\prime}(X(t))(\zeta(t))+\frac{1}{2} \operatorname{TRACE} \theta^{\prime \prime}(X(t)) \circ[\tilde{\xi}(t) \times \tilde{\xi}(t)]\right\} d t .
\end{aligned}
$$

THEOREM 3. Let $f$ and $g$ be maps from $\left[t_{0}, \infty\right) \times L(H ; H) \times \Omega$ into $L^{3}(B ; R)$ and $L(H ; H)$, respectively $\left(t_{0} \geqq 0\right)$. Assume that $f$ and $g$ satisfy the following conditions:

(i) for each $S \in L(H ; H), f(\cdot, S, \cdot)$ and $g(\cdot, S, \cdot)$ are nonanticipating;

(ii) there is a constant $c$ such that, with probability 1 ,

$$
\|f(t, S)-f(t, T)\|_{2}+\|g(t, S)-g(t, T)\|_{H ; H} \leqq c\|S-T\|_{H ; H},
$$

and

$$
\|f(t, S)\|_{2}^{2}+\|g(t, S)\|_{H ; H}^{2} \leqq c\left(1+\|S\|_{H ; H}^{2}\right)
$$

for all $t \in\left[t_{0}, \infty\right)$ and $S, T \in L(H ; H)$.

Then the $L(H ; H)$-valued stochastic differential equation

$$
d Y(t)=f(t, Y(t)) d W(t)+g(t, Y(t)) d t
$$

has a unique nonanticipating continuous solution. The solution is a Markov process.

4. An application. If $S \in L(H ; H)$ and $T \in L^{3}(H ; R)$ then we define $S \triangle T \in L^{3}(H ; R)$ by $(S \triangle T) \sim(x)=S \circ(\widetilde{T}(x)), x \in H$. Consider the stochastic integral equation

$$
X(t)=I+\int_{0}^{t} X(s) \Delta \xi(s) d W(s)+\int_{0}^{t} X(s) \circ \eta(s) d s,
$$

where $\xi$ and $\eta$ are bounded nonanticipating processes with state spaces $L^{3}(B ; R)$ and $L(H ; H)$, respectively.

THEOREM 4(GIRSANOV-SKOROKHOD-MCKEAN'S FORMULA). Suppose that, with probability $1,\{\tilde{\xi}(t)(x), \eta(t) ; 0 \leqq t<\infty, x \in B\}$ forms a commutative family of operators. Then the solution of the above equation can be represented by

$$
X(t)=\exp \left\{\int_{0}^{t} \xi(s) d W(s)+\int_{0}^{t}\left\{\eta(s)-\frac{1}{2} \operatorname{TRACE}(\kappa \circ[\tilde{\xi}(s) \times \tilde{\xi}(s)])\right\} d s\right\},
$$


where $\kappa$ is the map from $L(H ; H) \times L(H ; H)$ into $L(H ; H)$ given by $\kappa(S, T)$ $=S \circ T$.

\section{REFERENCES}

1. L. Gross, Abstract Wiener spaces, Proc. Fifth Berkeley Sympos. Math. Statist. and Probability (Berkeley, Calif., 1965/66), vol. II : Contributions to Probability Theory, part 1, Univ. of California Press, Berkeley, Calif., 1967, pp. 31-42. MR 35 \# 3027.

2. K. Ito, On a formula concerning stochastic differentials, Nagoya Math. J. 3 (1951), 55-65. MR 13, 363.

3. K. Ito and M. Nisio, On the convergence of sums of independent Banach space valued random variables, Osaka J. Math. 5 (1968), 35-48. MR 38 \# 3897.

4. D. Kannan and A. T. Bharucha-Reid, An operator-valued stochastic integral, Proc. Japan Acad. 47 (1971), 472-476.

5. H. H. Kuo, Stochastic integrals in abstract Wiener space, Pacific J. Math. 41 (1972), $469-483$.

6. Diffusion and Brownian motion on infinite dimensional manifolds, Trans. Amer. Math. Soc. 169 (1972), 439-459.

7. _ - Stochastic integrals in abstract Wiener space. II: Regularity properties (in preparation).

8. H. H. Kuo and M. Ann Piech, Stochastic integrals and parabolic equations in abstract Wiener space, Bull. Amer. Math. Soc. 79 (1973) (to appear).

9. H. P. McKean, Stochastic integrals, Probability and Math. Statist., no. 5, Academic Press, New York, 1969. MR 40 \#947.

Department of Mathematics, University of Virginia, Charlottesville, Virginia 22903 